\title{
THE LIDOS.RFQ.DESIGNER DEVELOPMENT
}

\author{
Boris Bondarev, Alexander Durkin, Yuri Ivanov, Igor Shumakov, Stanislav Vinogradov \\ Moscow Radiotechnical Institute RAS, \\ 132, Warshavskoe shosse, Moscow, Russia, 113519 lidos@aha.ru \\ Alexander Ovsyannikov, Dmitri Ovsyannikov \\ Saint-Petersburg State University, \\ Bibliotechnaya square, 2, Petrodvorets, St.Petersburg, Russia, 198904 dovs@apcp.apmath.spbu.ru
}

\section{Abstract}

The LIDOS.RFQ.Designer code package gives users the possibility to proceed successfully from input data up to RFQ channel design and space-chargedominated beam simulation. The code main feature is a maximum of scientific visualization for each calculation step. The package contains codes with three levels of mathematical model complexity. The first-level codes make not only a preliminary choice of the main parameter arrays on the basis of a simplified physical model but also chosen design optimization. In the case of high-current CW RFQ radiation purity (minimum of lost particles) is considered as the main optimization criteria. The secondlevel codes are used for RF field calculations taking into account the real shape of the RFQ vanes. The third-level codes are based on complex PIC-models that are needed for beam simulation in the chosen channel version.

\section{INTRODUCTION}

The LIDOS.RFQ.Designer code package is best demonstrated in the report [1]. The code development is performed in two directions:

1) provision is made for different ion types concurrently simulation, including concurrently simulation of beams with opposite charge sign;

2) linking together LIDOS ADVISOR (the first level code) with code package BDO RFQ* worked out in St.Petersburg University. It gives a possibility to use mathematical optimization methods during the channel calculation procedure.

Concurrently simulation of different ion types is topical for generation of advanced facility for the production of nuclei far from stability [2]. Optimization problems are currently central generation superpower accelerations for ADT applications, as example, which must be practically free of beam losses.

\footnotetext{
* The code package BDO RFQ is supported in part by the Russian Foundation for Basic Research, project 99-0100678.
}

\section{DIFFERENT ION TYPE SIMULATION}

Provision is made for beam simulation codes by PIC methods (the three level) to preset input beam parameters for different ion types (ion mass and charge, ion current, macro-particle number and so on. As a result user has cartoon that demonstrates beam parameter variation during acceleration process as well as beam output characteristics for each ion types.

\section{BDO-RFQ CODE}

New approaches to the solving of optimization problems for charged particles dynamics in accelerators were developed in the following works [3-7]. They include: construction of mathematical model of controlled dynamical process; choice of control functions or parameters of optimization; construction of quality functionals, which allow efficient evaluation of various characteristics of examined controlled motions; analytical representation of the functionals variations, which allow to construct various methods of optimization for quality functionals; construction of methods and algorithms of optimization.

The complex of programs for modeling and optimization of dynamics of charged particles in RFQ structure is developed. The complex of programs is supposed to allow the usage of various beam dynamics models in the process of RFQ structure optimization. Particular attention was paid to visualization of modeling and optimization processes and to creation of userfriendly interface.

The complex of programs is structurally divided into controlling cover and blocks, which describe various models of particles' dynamics. With this various models are described as special procedures containing all necessary information about particular model like parameters of the model, equations of the motion, algorithms of quality functionals calculation, the procedure of re-calculation control parameters of one model of dynamics into another, the range of values (characterizing the dynamics) put on the display etc.

Given approach to the construction of integrated system of modeling, optimization and visualization of charged particles dynamics in RFQ structure in its nature is like object-oriented programming. In this case it is 
possible to consider as objects models which describe dynamics, while uniting commanding shell realizes standard (typical) operations over models, i.e. choice of model, calculation of dynamics, optimization, visualization of dynamic process characteristics and a transition from one model to another.

Representation of control models as various separate blocks, possibility of translation of information between them and unified (united) system of model's control allow a realization of step by step optimization of such complicated object as the RFQ structure. With this it is natural to use simple models of dynamics on initial steps of optimization and on follows steps of it more developed models are necessary for verification of results and for further optimization.

Under consideration of longitudinal motion optimization criteria are the following: obtaining of maximal capture of particles into acceleration mode; obtaining of required or maximal possible output energy; minimization of defocusing factor effect; obtaining of bunching monotonicity.

Problems of transverse motion optimization are considered too. Conducted calculations have shown the effectiveness of step-by-step optimization on various stages of the process of the optimization. Thus the minimization of the defocusing factor on the stage of the optimization of longitudinal motion allows the focusing of the beam with the conservation of chosen dynamics of longitudinal motion

The library of models can be added by new models. For this purpose standard mechanism of description of new model and its inclusion to optimization shell is developed.

\section{NUMERICAL SIMULATION}

The code tools described above were used for RFQ designing. Main results are presented below to demonstrate power new code BDO-RFQ.

The following parameters were preset:
Accelerating Particles
Protons
Input Energy
Output Energy
$0.095 \mathrm{MeV}$
Beam Current
$5 \mathrm{MeV}$
$100 \mathrm{~mA}$
Beam Emittance
$0.15 \pi \mathrm{cm} \cdot \mathrm{mrad}$
Operating Frequency
$352 \mathrm{MHz}$

There were some restrictions and requirements:

Length

Beam Transmission

no more than $8 \mathrm{~m}$

no less then $98 \%$

Defocusing factor

no more than 0.01

In figures 1-6 variations of some characteristics are shown for several RFQ structures [8-10]:

I - LEDA-RFQ (USA);

II $\quad-$ IPHI-RFQ (France);

III, IV - versions of RFQ structures found with Code BDO-RFQ (Russia).

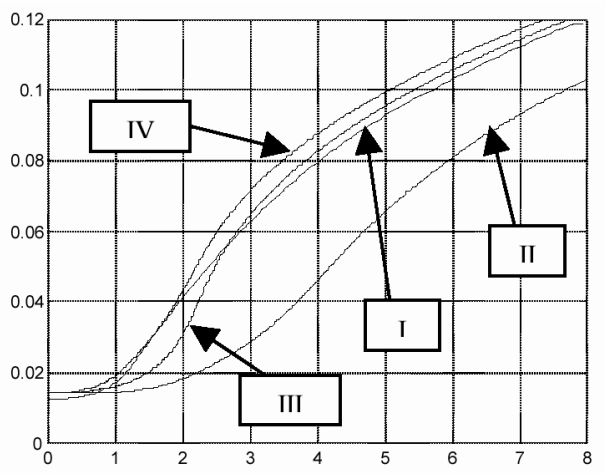

Fig.1. Synchronous particle reduced velocity vs. Z.

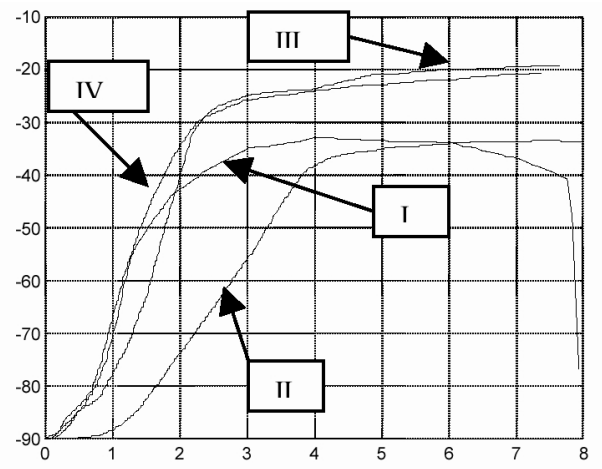

Fig.2. Synchronous phase vs. $Z$.

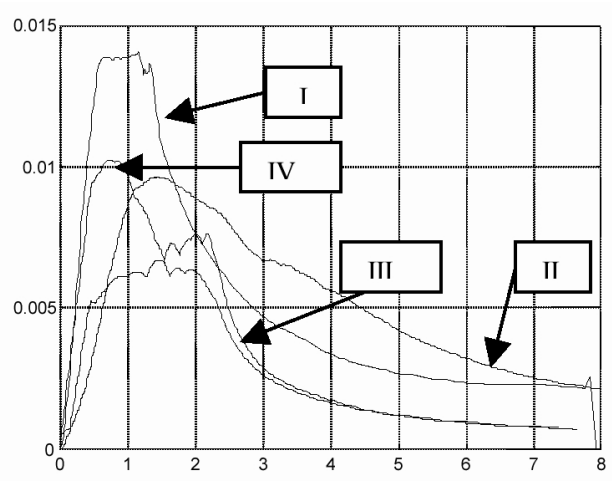

Fig.3. Defocusing factor vs. $Z$.

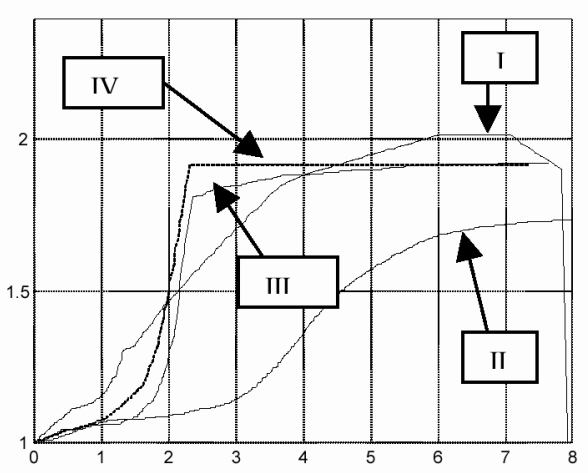

Fig.4. Vane modulation vs. Z. 


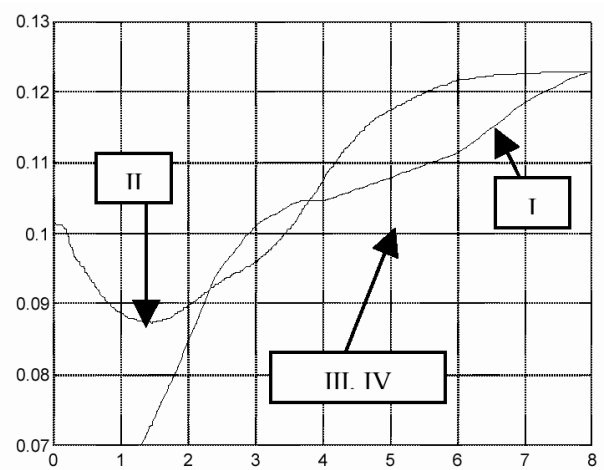

Fig.5. Intervane voltage vs. Z.

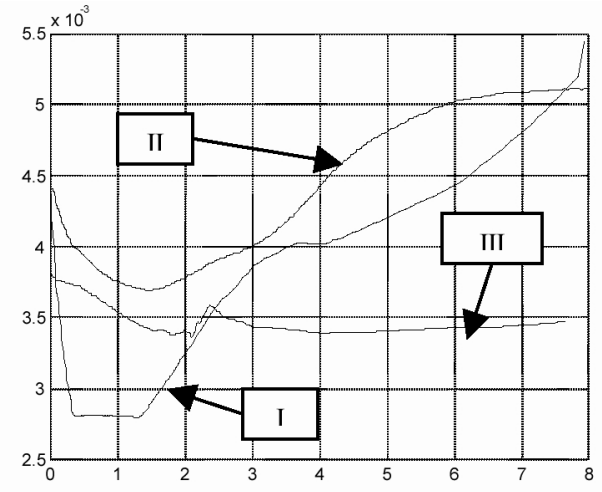

Fig.6. Beam average radius vs. $Z$.

The aim of optimization in variants III and IV was to get accelerator structure with output energy $5 \mathrm{MeV}$ with minimal length. With this in variant III more strict limits on change of defocusing factor had been taken into account.

Further, both variants III and IV had been calculated to output energy $6.7 \mathrm{MeV}$. During analyzing and optimization of transverse motion it was suppose that intervane voltage is equal to $100 \mathrm{kV}$.

At present time code package for RFQ optimization is generated in the frame of ISTC Project \# 912. In future it will insert into LIDOS.RFQ.

\section{REFERENCES}

1. Bondarev B.I., Durkin A.P., Vinogradov S.V., Shumakov I.V. "New Tasks and New Codes for RFQ Beam Simulation", Proceedings LINAC'2000, p.830-832.

2. Ostroumov P.N., Shepard K.W. "Multiple-charge Beam Dynamics in an Ion Linac" Physical Review V.3 030101 (2000).

3. Bondarev B.I., Durkin A.P., Ovsyannikov A.D., "New Mathematical Optimization Models for RFQ Structures", Proceedings of the 1999 Particle Accelerator Conference, New York, 1999, pp. 2808-2810.

4. Ovsyannikov A.D., "New Approach to Beam Dynamics Optimization Problem ", Proceedings of the 6th International Computational Accelerator Physics Conference ICAP 2000, Darmstadt, Germany, 2000.

5. Ovsyannikov D.A., "Modelling and Optimisation of Charged Particle Beam Dynamics", Leningrad State University, Leningrag, 312p., (1990) (in Russian).

6. Bondarev B.I., Durkin A.P., Vinogradov S.V., Ovsyannikov D.A., Ovsyannikov A.D., "RFQ Optimisation: Methods \& Codes", Proceedings of the 6-th International Computational Accelerator Physics Conference ICAP 2000, Darmstadt, Germany, 2000.

7. Ovsyannikov D.A., "Modeling and Optimization Problems of Charged Particle Beams Dynamics", Proceedings of the $4^{\text {th }}$ European Control Conference, Brussels, 1997, pp. 390-394.

8. R.Ferdinand, J-M.Lagniel, P.Mattei, S.Nath "Optimization of RFQ Design", Proceeding of EPAC-98 Conference, June 22-26, Stockholm, Sweden, pp.1106-1108

9. D.Schrage, et al., "CW RFQ Fabrication and Engineering", Proceedings of LINAC98, Chicago, 1998, pp.679-683.

10. W.P.Lysenko, et al., "High Energy Beam Transport Beam Line for LEDA", Proc. LINAC98, Chicago, 1998, pp.496-498 